\title{
Food-reinforced visual discrimination in rats with anterior or posterior amygdaloid lesions
}

\author{
WILLIAM L. STOLLER and RITA A. V. STOLLER \\ Indiana University at Kokomo, Kokomo, Indiana
}

\begin{abstract}
Albino rats were trained on a visual discrimination task for which leverpressing in the presence of a cuelight was continuously reinforced with food. Upon attainment of criterion, the rats received bilateral electrolytic lesions in either the anterior or the posterior amygdala, or were subjected to a corresponding control operation. For both placement sites, the discriminative behavior of the lesioned animals, as well as their activity in an open field, was comparable to that of the corresponding controls. The results are discussed in terms of possible excitatory and inhibitory amygdaloid influences on appetitively motivated instrumental behavior and are interpreted in relation to previous research. It is proposed that the middle portion of the amygdala as a whole may exert an excitatory influence on appetitive arousal.
\end{abstract}

The experimental literature shows that the amygdaloid complex has region-dependent functions. In cats, for example, acquisition of active-avoidance behavior has been impaired by lesions in the rostral part of the lateral amygdaloid nucleus, whereas a passive-avoidance deficit has been associated with damage to the medial nucleus (Ursin, 1965). It has been observed that, in dogs, deficient instrumental performance resulting from lesions of the dorsomedial amygdala can be improved by lesions of the lateral amygdala (Fonberg, 1975). Pellegrino (1968) found that rats with bilateral lesions of the basolateral portion of the amygdala were impaired in passive avoidance, differential reinforcement of low rates (DRL) performance, and spatial alternations without cues, whereas animals with lesions in the corticomedial region showed no impairments in these tasks other than a small deficiency in passive avoidance. Henke (1974) observed that medial lesions produced an earlier breakdown of responding on progressively increasing fixed-ratio schedules of reinforcement, whereas lateral lesions produced response perseveration. Kemble and Godding (1981), also using rats, found that corticomedial lesions attenuated grooming behavior induced by limited water access but increased grooming induced by water spray. In contrast, basolateral lesions had no consistent influence on grooming.

In a previous study conducted in this laboratory (Stoller, 1972), relatively large bilateral lesions in the amygdaloid complex of rats reduced responding on a food-reinforced visual discrimination task under conditions of both reinforcement and nonreinforcement. In view of findings such as those above and evidence of region-dependent amygdaloid influences on ingestive behavior (Grossman, 1972),

This study was partially supported by funds received from the Office of Research and Advanced Studies at Indiana University. Requests for reprints should be sent to William L. Stoller, Indiana University at Kokomo, 2300 South Washington Street, Kokomo, IN 46902. in the present study we sought to extend the analyses of Stoller's investigation to a comparison of the possible effects of anterior and posterior amygdaloid lesions on foodreinforced visual discriminative behavior.

\section{METHOD}

\section{Subjects}

Thirty-two male albino rats obtained from the Holtzman Company (Madison, WI) were used. The animals were about 120 days old at the beginning of the experiment and had no previous experimental history. They were maintained in individual cages.

\section{Apparatus}

A $27.9 \times 20.3 \times 20.3 \mathrm{~cm}$ (inside dimensions) operant chamber housed in a sound-attenuation box was used in training and testing the subjects. The front and rear walls of the chamber were constructed from $0.1-\mathrm{cm}$ stainless steel; the sides and hinged top were made of $0.6-$ and $0.4-\mathrm{cm}$ Plexiglas, respectively. The floor consisted of $0.5-\mathrm{cm}$ stainless steel rods spaced $1.6 \mathrm{~cm}$ center to center. A single lever, which extended $5.1 \mathrm{~cm}$ across the front wall and was $0.1 \mathrm{~cm}$ thick, projected $2.5 \mathrm{~cm}$ into the chamber. The lever was $6.5 \mathrm{~cm}$ above the grid floor and required a force of $15 \mathrm{~g}(0.3-\mathrm{cm}$ excursion) to activate an attached microswitch. A cuelight was centered $6.5 \mathrm{~cm}$ above the lever. Reinforcements for leverpressing, which consisted of single 45-mg food pellets (P. J. Noyes), were delivered from a pellet dispenser into a food cup located in the lower right corner of the front wall, $2.2 \mathrm{~cm}$ above the floor. Leverpresses were recorded on a manually resettable four-digit counter.

An unpainted box with a $91.4 \times 91.4 \mathrm{~cm}$ floor and $45.7-\mathrm{cm}$-high sides (inside dimensions) was used in obtaining open-field activity measures for the animals. The floor and sides were made of $1.3-\mathrm{cm}$ plywood; the hinged top consisted of a piece of $0.2-\mathrm{cm}$ Plexiglas held in a frame of $1.9-\mathrm{cm}$ plywood. The floor was divided into 36 equal squares.

\section{Procedure}

The procedure was based in part on an experimental design used by Schwartzbaum, Kellicutt, Spieth, and Thompson (1964). The rats were first adapted to a 20 -h food deprivation schedule over a 5-day period. Each animal was then given daily 8-min sessions during which it was trained to press the lever for food under conditions of continuous reinforcement and randomly presented intervals of cuelight on and cuelight off. Discrimination training began on the day following the day on which an animal had made a total of 50 responses over all such sessions. S+ was cuelight on, and $\mathrm{S}-$ was cuelight off. For $\mathrm{S}+$, all leverpresses were 
reinforced, whereas for $\mathrm{S}-$, leverpresses were never reinforced. $\mathrm{S}+$ and $S-$ were each presented twice for periods of 2 min during daily 8-min discrimination sessions. The order of presentation was random. An animal received discrimination training until (1) the mean number of responses per minute during $\mathrm{S}+$ was at least three times as great as that during $\mathrm{S}$ - over eight consecutive sessions, and (2) this ratio was attained for at least five of the eight sessions.

There were 8 randomly assigned subjects for each of four treatments: anterior amygdaloid lesion (AN-AM), anterior amygdaloid control (AN-AM-CON), posterior amygdaloid lesion (POS-AM), and posterior amygdaloid control (POS-AM-CON). An animal underwent surgery upon attainment of criterion. A 4-day recovery period followed, during which food and water were provided ad lib. When the animal had again been adapted to the 20-h food deprivation schedule over 5 days, testing for retention of the discrimination was conducted. The requirements for criterion performance were the same as the preoperative requirements. Criterion performance was followed by two 8-min extinction sessions, given on successive days, during which $\mathrm{S}+$ was continuously present.

Activity was assessed for each of the subjects immediately before each postoperative lever-box session. An animal was placed on the floor of the plywood and Plexiglas box and was then allowed to move about the enclosure freely for $15 \mathrm{~min}$. An observer who was unaware of the treatment to which the rat had been assigned recorded the number of squares entered by the animal during successive 5-min periods. An animal was scored as having entered a given square if it placed all four feet within the square. The measures were obtained with the box under normal room illumination.

Analyses of variance were used in the statistical evaluation of the activity data. Two-tailed $t$ tests and two-tailed Mann-Whitney $U$ tests, in addition to analyses of variance, were applied to the data on lever-box performance.

\section{Surgery and Histology}

Bilateral electrolytic lesions were placed in the amygdala with the aid of a Baltimore stereotaxic instrument. A 1-mA anodal current was passed for $12 \mathrm{sec}$ through a steel needle $(0.5 \mathrm{~mm}$ diam) that was insulated except for the tip. The coordinates for anteriorly placed lesions were $2.0 \mathrm{~mm}$ posterior to bregma, $4.0 \mathrm{~mm}$ lateral to the midline, and $8.0 \mathrm{~mm}$ below the dura mater. The coordinates for lesions produced in the posterior amygdala differed from these only in that the anterior-posterior (AP) position was $3.8 \mathrm{~mm}$ posterior to bregma. Both sets of coordinates were based on an examination of pilot lesions. The incisor bar was positioned $8 \mathrm{~mm}$ below the interaural line. Animals that received control operations underwent the same surgical procedures as those in the corresponding lesion groups, except that no current was applied. All animals were maintained under sodium pentobarbital anesthesia (Nembutal, $60 \mathrm{mg} / \mathrm{kg}$ i.p.) during surgery.

The rats were killed with sodium pentobarbital at the end of the experiment, and the brains were fixed in $10 \%$ Formalin. Representative $40-\mu$ coronal sections obtained through the use of a frozen tissue technique were mounted and stained with cresyl violet. The lesions were evaluated with the aid of the König and Klippel (1963) atlas.

\section{RESULTS}

\section{Behavioral Data}

Figure 1 shows postoperative discrimination performance in terms of percentage changes in response rate under both $\mathrm{S}+$ and $\mathrm{S}-$ for the four treatment groups. Separate sets of $t$ tests were performed on the data of Sessions 1-4 and 5-8.

During the first four sessions under $\mathrm{S}+$, responding of the AN-AM group did not differ significantly from that of the AN-AM-CON group, nor did the responding of the POS-AM group differ significantly from that of the

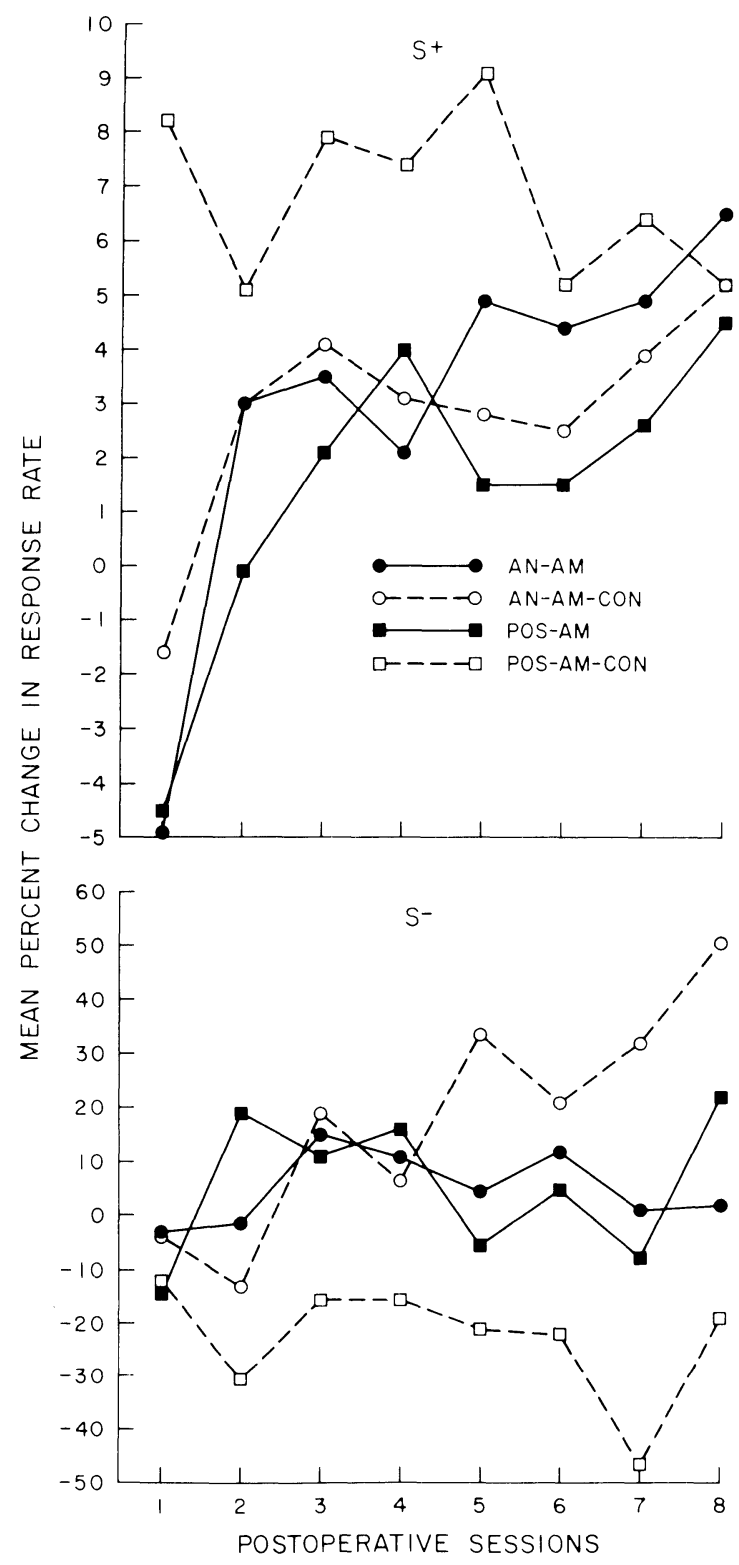

Figure 1. Postoperative changes in response rate for the discrimination task under conditions of reinforcement $(\mathrm{S}+)$ and nonreinforcement $(\mathrm{S}-$ ). Baselines provided by performance during last five preoperative sessions. $\mathbf{A N}-\mathbf{A M}=$ anterior amygdaloid lesion, AN-AM-CON = anterior amygdaloid control, $\mathbf{P O S}-\mathbf{A M}=$ posterior amygdaloid lesion, POS-AM-CON = posterior amygdaloid control.

POS-AM-CON group $[t \mathrm{~s}(14)=-0.39$ and -1.51 , anterior and posterior lesion analyses, respectively; $p s>.05]$. The results for the second block of four sessions under $\mathrm{S}+$ were consistent with those for the first. Similarly, for the first four postoperative sessions under $\mathrm{S}-$, the performance of the AN-AM group did not differ significantly from that of the AN-AM-CON group, nor did the performance of the POS-AM group differ significantly from that of the POS-AM-CON group [ts $(14)=$ 0.22 and 1.72 , anterior and posterior lesion analyses, 
respectively; $p s>.05]$. The results for the second block of four sessions under $\mathrm{S}-$ agreed with those for the first. Neither lesion group differed significantly from the corresponding control group in terms of the number of postoperative sessions required to regain criterion $(U s=$ 30 and 44 for anterior and posterior lesion analyses, respectively; $p s>.05)$.

Figure 2 summarizes performance of the groups during each of the two extinction sessions. Response rates generally decreased over each session. For Session 1, analyses of variance on data over the extinction interval yielded a significant minutes effect both for AN-AM versus AN-AM-CON and for POS-AM versus POS-AM-CON $[F(3,42)=23.7, p<.001$, and $F(3,42)$ $=19.6, p<.001$, respectively]. However, the treatment effects for AN-AM versus AN-AM-CON and POS-AM versus POS-AM-CON were nonsignificant, as were the treatments $\times$ minutes interactions $(F$ tests, $p s>.05)$. The results for Session 2 were parallel to those for Session 1.

Figure 3 summarizes data on activity of the four groups preceding postoperative lever-box Sessions 1-2 and 9-10. In both the initial and the later sessions, activity generally decreased over the 15-min period. For Sessions 1-2, analyses of variance on data over the observation interval yielded a significant minutes effect both for AN-AM versus AN-AM-CON and for POS-AM versus POS-AM-CON $[F(2,28)=63.1, p<.001$, and $F(2,28)$ $=127.4, p<.001$, respectively]. The treatment effects for the anterior and posterior lesion analyses were non-

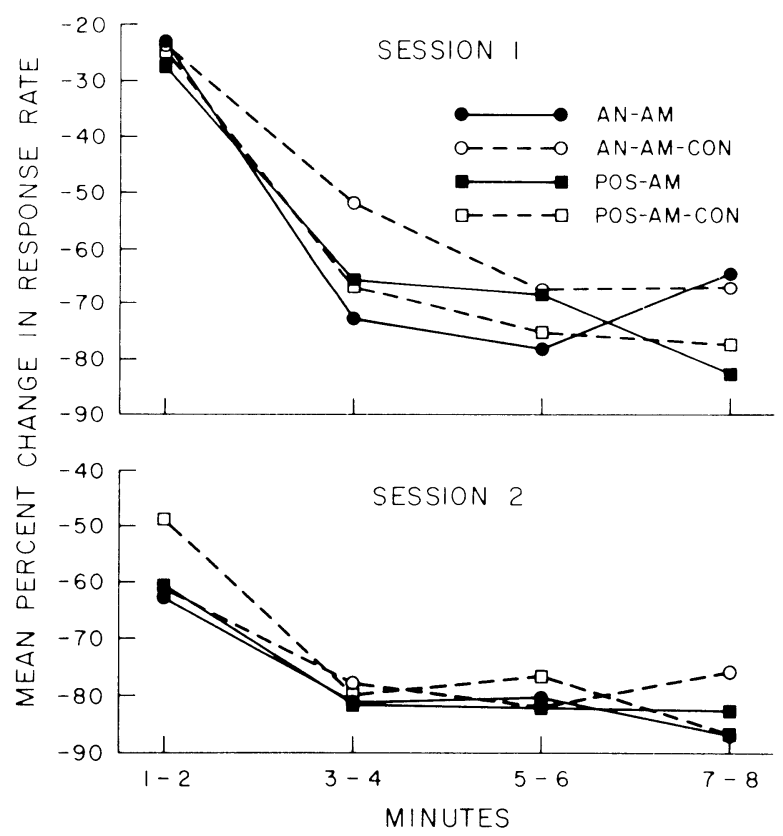

Figure 2. Changes in response rate for the discrimination task during each of two extinction sessions. Baseline provided by performance under $\mathbf{S}+$ during last five preextinction sessions. Abbreviations as for Figure 1.

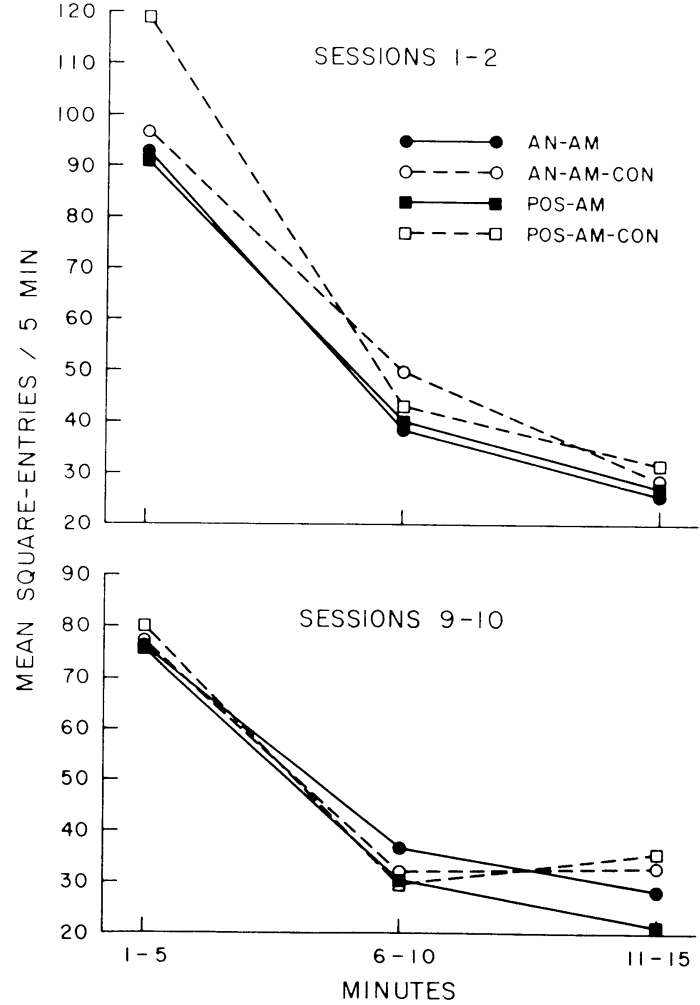

Figure 3. Activity preceding postoperative lever-box Sessions 1-2 and 9-10. Abbreviations as for Figure 1.

significant, as was the treatments $\times$ minutes interaction for AN-AM versus AN-AM-CON ( $F$ tests, $p$ s $>.05$ ). For POS-AM versus POS-AM-CON, however, the treatments $\times$ minutes interaction was significant $[F(2,28)=$ $3.8, p<.05]$. An examination of Figure 3 indicates that the latter interaction may be attributable to the fact that less activity was displayed by the animals with posterior lesions than by their controls during the first $5 \mathrm{~min}$. The results for Sessions 9-10 were parallel to those for Sessions $1-2$, except that the treatments $\times$ minutes interaction for POS-AM versus POS-AM-CON was nonsignificant $(F$ test, $p>.05)$.

\section{Lesion Placements}

The anterior lesions typically included nearly all of the anterior amygdaloid area. At the AP plane where the damage tended to be greatest, corresponding approximately to A $6650 \mu$ in the König and Klippel (1963) atlas, the lesions extended about $1.5 \mathrm{~mm}$ mediolaterally and $1.7 \mathrm{~mm}$ dorsoventrally. The lesions were usually about $1.5 \mathrm{~mm}$ in anteroposterior extent. Minor damage occurred to structures lying outside of the amygdaloid region.

The posterior lesions typically included much of the corticomedial and basolateral regions, which showed essentially the same degree of damage, and tended to be maximal at an AP plane corresponding approximately to A $3900 \mu$ in the König and Klippel (1963) system. At this 
plane the lesion measurements were about 1.5 and $1.3 \mathrm{~mm}$ in the mediolateral and dorsoventral dimensions, respectively. The lesions measured $1.5 \mathrm{~mm}$ anteroposteriorly. Negligible damage occurred to extra-amygdaloid structures.

\section{DISCUSSION}

In the present study, food-reinforced visual discriminative behavior of rats with anterior or posterior amygdaloid lesions was comparable to that of corresponding operated control animals. In addition, openfield activity of the lesioned animals was generally similar to that of their controls.

Grossman (1972) pointed out that the amygdaloid complex appears to exert both excitatory and inhibitory influences on feeding and feedingrelated activities. Excitatory influences seem to arise mainly from the dorsal, anterior, and medial aspects of the complex, whereas inhibitory influences apparently originate primarily in the ventral, posterior, and lateral regions. If the anterior amygdala, as a whole, exerts an excitatory influence on appetitively motivated instrumental behavior, possibly through modulation of a hypothalamic arousal mechanism, one might expect damage in this region to diminish such behavior under $\mathrm{S}+$ and, to a lesser extent, under S- . If the posterior amygdala, on the other hand, essentially inhibits this form of behavior, perhaps through suppression of an excitatory mechanism in the basomedial hypothalamus (Mabry \& Campbell, 1975), it might be expected that damage in this portion of the complex would increase responding under these conditions. Furthermore, a food-reinforced barpress response should be less resistant to extinction as a consequence of anterior damage, whereas damage to the posterior portion of the structure should result in increased resistance to extinction. Open-field activity of unsated subjects similarly should be reduced by anterior damage and should be increased by damage to the posterior portion of the complex. However, the comparability of performance of the lesioned and control animals in this study may reflect the removal, from both the anterior and posterior amygdala, of approximately equal proportions of excitatory and inhibitory influences on appetitive arousal. The spatial distribution of such influences, it should be noted, may be somewhat different from that of influences on feeding (Mabry \& Campbell, 1975).

In a previous study utilizing a design and techniques similar to those used in the present investigation, bilateral lesions located essentially in the anterior-middle region of the amygdala reduced responding under conditions of both reinforcement and nonreinforcement (Stoller, 1972). The current anterior lesion data, together with this previous observation, suggest that the middle portion of the amygdala as a whole exerts an excitatory influence on appetitive arousal.

\section{REFERENCES}

FoNBERG, E. (1975). Improvement produced by lateral amygdala lesions on the instrumental alimentary performance impaired by dorsomedial amygdala lesions in dogs. Physiology \& Behavior, 14, 711-717.

GrossmaN, S. P. (1972). Neurophysiologic aspects: Extrahypothalamic factors in the regulation of food intake. Advances in Psychosomatic Medicine, 7, 49-72.

Henke, P. G. (1974). The effect of medial and lateral lesions in the amygdala on fixed-ratio performance. Canadian Journal of Psychology, 28, 245-251.

Kemble, E. D., \& Godding, P. R. (1981). Effects of basolateral or corticomedial amygdaloid lesions on grooming, consummatory, and locomotor behaviours in rats. Behavioural Processes, 6, 161-169.

KöNIG, J. F. R., \& KLIPPEL, R. A. (1963). The rat brain. Baltimore: Williams \& Wilkins.

MABRY, P. D., \& CAMPBELl, B. A. (1975). Food-deprivation-induced behavioral arousal: Mediation by hypothalamus and amygdala. Journal of Comparative \& Physiological Psychology, 89, 19-38.

Pellegrino, L. (1968). Amygdaloid lesions and behavioral inhibition in the rat. Journal of Comparative \& Physiological Psychology, 65, 483-491.

Schwartzbaum, J. S., Kellicutt, M. H., Spieth, T. M., \& ThompSON, J. B. (1964). Effects of septal lesions in rats on response inhibition associated with food-reinforced behavior. Journal of Comparative \& Physiological Psychology, 58, 217-224.

StOLLER, W. L. (1972). Effects of septal and amygdaloid lesions on discrimination, eating and drinking. Physiology \& Behavior, 8 , 823-828.

URSIN, H. (1965). Effect of amygdaloid lesions on avoidance behavior and visual discrimination in cats. Experimental Neurology, 11, 298-317.

(Manuscript received for publication August 7, 1987.) 